Revue internationale P.M.E.

Économie et gestion de la petite et moyenne entreprise

Revure

internationale

PME

\title{
PME, mondialisation et stratégies
}

\section{Georges Fernandez et Alain Noël}

Volume 6, numéro 3-4, 1994

URI : https://id.erudit.org/iderudit/1008235ar

DOI : https://doi.org/10.7202/1008235ar

Aller au sommaire du numéro

Éditeur(s)

Presses de l’Université du Québec

ISSN

0776-5436 (imprimé)

1918-9699 (numérique)

Découvrir la revue

Citer cette note

Fernandez, G. \& Noël, A. (1994). PME, mondialisation et stratégies. Revue internationale P.M.E., 6(3-4), 145-163. https://doi.org/10.7202/1008235ar

\section{Résumé de l'article}

Le contexte de mondialisation des affaires a forcé les multinationales à accentuer leur course vers les économies d'échelle, les stratégies de coût minimal et l'exploitation de toutes les opportunités d'affaires à travers le monde. Par ailleurs, peu de choses ont été dites quant aux modes de réaction des PME face à cette invasion de leur territoire par les grandes entreprises. Dans cet article, nous nous penchons sur les conséquences de la mondialisation pour des PME dont l'activité est essentiellement nationale et n'ayant a priori ni l'infrastructure ni les ressources pour affronter ce phénomène. Notre analyse soutient que ces petites entreprises peuvent envisager plusieurs options intéressantes notamment via les alliances stratégiques. Si un tel recours présente un potentiel séduisant, nous soulignons par ailleurs que leur mise en œuvre sera ardue et parfois impossible pour les entreprises qui tiendront à préserver à tout prix leur autonomie. Quant aux solutions, elles divergent selon quelques cas d'espèce que nous décrivons. 


\title{
PME, mondialisation et stratégies
}

\author{
Georges FERNANDEZ* \\ Alain NOËL**
}

\begin{abstract}
RÉSUMÉ
Le contexte de mondialisation des affaires a forcé les multinationales à accentuer leur course vers les économies d'échelle, les stratégies de coût minimal et l'exploitation de toutes les opportunités d'affaires à travers le monde. Par ailleurs, peu de choses ont été dites quant aux modes de réaction des PME face à cette invasion de leur territoire par les grandes entreprises. Dans cet article, nous nous penchons sur les conséquences de la mondialisation pour des PME dont l'activité est essentiellement nationale et n'ayant a priori ni l'infrastructure ni les ressources pour affronter ce phénomène. Notre analyse soutient que ces petites entreprises peuvent envisager plusieurs options intéressantes notamment via les alliances stratégiques. Si un tel recours présente un potentiel séduisant, nous soulignons par ailleurs que leur mise en cuvre sera ardue et parfois impossible pour les entreprises qui tiendront à préserver à tout prix leur autonomie. Quant aux solutions, elles divergent selon quelques cas d'espèce que nous décrivons.
\end{abstract}

\begin{abstract}
Globalization of business has pushed multinational corporations into a race for economies of scale and total cost leadership strategies, thus trying to take advantage of all available business opportunities worldwide. Not much has been said on the other hand about how small businesses should react to this invasion of their local markets by large global corporations. This article addresses the issues faced by small businesses who do not have the resources nor the infrastructure to deal with these new competitive pressures. Our analysis proposes that the small business firm can evaluate several options, namely strategic alliances, to face this problem. If alliance solutions are appealing, we argue that their implementation could prove difficult, if not impossible to those businesses who will try to entirely protect their autonomy. Three categories of cases and solutions are presented and discussed.
\end{abstract}

* Georges Fernandez est étudiant au programme de doctorat à l'École des Hautes Études Commerciales de Montréal. Adresse : CETAI, École des HEC, 5255, avenue Decelles, Montréal (Québec) H3T 1V6

** Alain Noël est professeur agrégé de stratégie et directeur associé du CETAI (Centre d'études en administration internationale) à l'École des Hautes Études Commerciales de Montréal. Adresse : CETAI, École des HEC, 5255, avenue Decelles, Montréal (Québec) H3T 1V6 


\section{RESUMEN}

El contexto de la mundialización ha forzado a las multinacionales a orientar su curso hacia las economías de escala, las estrategias de costo minimo y la explotación de todas las oportunidades de negocios a través del mundo. Por otro lado, poco se ha dicho sobre las formas de reacción de las pequeñas y medianas empresas (PyME) al ser invadido su territorio por las grandes empresas. Este artículo estudia los consecuencias de la mundialización para las PyME, cuya activadad es esencialmente nacional, y no disponen a priori ni de la infraestructura ni de los recursos para enfrentar este fenómeno. Nuestro análisis sugiere que esas pequeñas empresas pueden evaluar varias opciones interesantes por la vía del establecimiento de las alianzas estratégicas. Si esta posibilidad es sugerente, nosotros pensamos que su implantación podria ser difícil, y a veces imposible, para las empresas que intentan preservar su autonomía a cualquier precio. Finalmente, son presentados y discutidos tres tipos de caso y sus soluciones.

\section{Introduction}

Depuis le début des années 80 , nous avons assisté à la naissance du phénomène de la mondialisation et nous sommes aujourd'hui témoins de sa montée en puissance avec la mise en place d'importants regroupements de marchés à l'échelle continentale tels que l'Union européenne ou l'ALENA en Amérique du Nord. Les réponses des entreprises à ce phénomène devraient dépendre de leurs caractéristiques intrinsèques (taille, activités, position concurrentielle sur le marché ou type d'organisation) et des particularités de leur environnement. À l'heure actuelle, on a surtout étudié la mondialisation dans la perspective des grandes multinationales en laissant souvent de côté ses graves répercussions pour les PME. Dans cet article, nous nous intéressons à cette nouvelle problématique posée aux petites et moyennes entreprises dont l'activité est essentiellement nationale. Ces entreprises locales, qui ne possèdent a priori ni l'infrastructure ni les ressources pour affronter les changements liés à la mondialisation, pourraient néanmoins se heurter à plus ou moins brève échéance aux enjeux que présente cette nouvelle réalité économique. Une réflexion stratégique permettant à ces PME de reconnaître les opportunités majeures tout comme les graves répercussions de la mondialisation s'impose aujourd'hui afin qu'elles puissent choisir, parmi les différentes réponses possibles à ce phénomène, celles qui permettront d'assurer leur croissance sinon, dans certains cas, leur survie.

Ainsi, dans cet article, nous analysons les différents éléments de solution auxquels ont accès ces entreprises lorsqu'elles sont en présence, dans leur environnement immédiat, d'entreprises concurrentielles d'envergure mondiale. Une telle analyse permet d'établir parmi les différentes options stratégiques qui leur sont offertes, celles qui pourront raisonnablement être envisagées. Comme 
nous voulons avant tout, par cette étude, soumettre le résultat d'une réflexion générale sur la problématique de la mondialisation pour l'ensemble des PME, sans pour autant s'attarder à des secteurs industriels précis, il est possible que certaines caractéristiques d'une industrie particulière puissent, dans certains cas, modifier les résultats de l'analyse proposée.

Dans la première partie de ce texte, nous nous pencherons sur le phénomène de la mondialisation pour pouvoir en déterminer les principales conséquences sur les PME. Les deuxième et troisième parties seront consacrées respectivement à l'identification et à l'évaluation des différentes options stratégiques que ces entreprises pourraient envisager. Nous conclurons enfin sur l'urgence de donner de nouveaux moyens aux PME pour qu'elles puissent mieux répondre à cette nouvelle problématique du marché.

\section{La mondialisation et ses conséquences pour les PME}

Nous allons, dans un premier temps, tenter de dégager les principaux concepts développés par certains auteurs qui se sont intéressés à la mondialisation afin de saisir la portée de ce phénomène. Par la suite, nous essaierons de relever les stratégies préconisées par les entreprises d'envergure mondiale ainsi que de prévoir leur comportement sur le marché.

L'analyse historique de la mondialisation menée par Chandler (1986) nous indique que ce phénomène constitue la suite des événements marquant l'évolution des grandes firmes qui sont passées d'entreprises nationales à des multinationales intégrées puis diversifiées pour devenir aujourd'hui des entreprises mondiales. De nos jours, les marchés n'offrent plus la croissance qu'ils ont connue jusqu'à la fin des années 60 ; pris individuellement, les marchés nationaux n'ont plus la capacité de soutenir la croissance de leurs entreprises. Ces dernières ont besoin d'un espace vital de plus en plus étendu et ne peuvent survivre qu'en ayant accès à des marchés de consommation de taille mondiale. En fait, la mondialisation est le résultat des profonds bouleversements de marchés auxquels on assiste sous le régime des négociations du GATT depuis 1950 et des importants changements technologiques, notamment, dans les domaines de l'information et de la communication. De ce fait, de grandes entreprises dominent à l'heure actuelle l'échiquier des marchés mondiaux.

Ohmae (1985) et Porter (1986) attribuent au phénomène de mondialisation des causes essentiellement économiques. Les conclusions de leurs travaux nous amènent à penser qu'il est essentiellement le résultat de trois principaux facteurs: 
1. L'homogénéisation des marchés due aux progrès réalisés dans les domaines de la communication et du transport: on constate que les consommateurs du monde entier ont de plus en plus les mêmes goûts et les mêmes besoins.

2. La course à l'innovation technologique caractérisée par une diminution rapide des cycles de vie des produits et pour laquelle les entreprises doivent réaliser d'importants investissements dans des programmes de recherche et développement afin de devancer la concurrence ou encore tout simplement pour rester dans la course.

3. L'obligation pour les entreprises d'investir constamment dans leur outil industriel afin de demeurer concurrentielles sur des marchés de plus en plus vastes et accessibles.

La conjugaison de ces trois facteurs pousse les entreprises mondiales à effectuer d'importants investissements ne pouvant être amortis, en raison de la diminution notable des cycles de vie des produits, que sur des périodes de courte durée. Cette nécessité d'amortir rapidement les capitaux investis dans l'outil industriel et la recherche et développement oblige les entreprises mondiales à se rabattre sur de gros volumes et, par conséquent, à s'attaquer à des marchés transnationaux. Dans ces grands marchés de masse, les entreprises mondiales auront comme principale préoccupation de constamment protéger et améliorer leur part de marché vis-à-vis de leurs concurrents et mettront tout en œuvre pour devenir les chefs de file incontestés des marchés locaux auxquels elles ont accès.

Parallèlement à ces grands marchés de masse homogènes, il existe aussi une tendance contradictoire dans les marchés, celle de l'hypersegmentation (Pickholz, 1988). En effet, on retrouve sur les marchés des demandes pour des produits ou des services très spécifiques et non standardisés qui créent des créneaux très étroits auxquels les grandes entreprises mondiales ayant misé sur les économies d'échelle n'ont pas accès. Ces micro-marchés pris individuellement offrent rarement un potentiel de croissance intéressant. Par contre, si l'on s'attaque à ces marchés de façon simultanée à l'échelle mondiale, ils représentent alors une opportunité de croissance pour les entreprises de moindre taille. Ainsi, il existe des PME à orientation mondiale qui pourraient bénéficier pleinement de l'ouverture des marchés pour attaquer sur leur terrain des PME locales confortablement installées dans des niches qu'elles croyaient jusqu'à présent inaccessibles.

En raison de la mondialisation, les PME locales devront, selon le cas, affronter soit de grandes multinationales d'envergure mondiale, soit d'autres PME déjà ouvertes à l'internationalisation et en voie de se mondialiser. Appelons PME mondiales les PME à orientation mondiale. 
Il est à présent essentiel d'établir quels sont les particularités et les avantages concurrentiels de ces firmes mondiales sur les PME locales. À partir des observations antérieures, nous pouvons émettre l'hypothèse que la production, la commercialisation, le développement technologique et les ressources financières seront les quatre principales caractéristiques qui, à notre avis, auront un poids déterminant dans un éventuel rapport de force entre ces différents types d'entreprise.

Un des gros avantages des «entreprises mondiales» est de pouvoir compter au niveau de l'exploitation sur une structure administrative permettant d'organiser leur production et leur approvisionnement sous forme de réseaux (Porter, 1986). Grâce à ces réseaux, tant les composants que les produits finis sont expédiés d'une unité de production spécialisée à une autre afin que chaque produit puisse par la suite être entièrement fabriqué dans une usine qui répondra à une demande mondiale. La configuration en réseaux permet à la fois de réaliser des économies d'échelle et de localisation. En effet, un réseau permet de produire des quantités optimales à l'endroit où un avantage au niveau des facteurs de production (main-d'œuvre ou matières premières) est présent. Ainsi, on peut surmonter les barrières tarifaires et non tarifaires tout en remplissant les exigences de contenu local. De plus, des économies d'expérience sont possibles, car chaque unité de production dispose des connaissances acquises par l'entreprise. Une PME mondiale ne bénéficiera que dans une bien moindre mesure de ces avantages, vu la petite taille de son infrastructure, alors que la PME locale ne connaîtra bien entendu aucun de ces avantages.

Quant à la commercialisation, les conditions locales des marchés priment souvent. Toutefois, la nature des produits et les caractéristiques des réseaux de distribution peuvent parfois avantager l'entreprise mondiale. En effet, pour les produits de consommation de masse, et particulièrement dans les pays où la distribution est bien organisée, la grande entreprise mondiale aura un avantage incontestable en ce qui concerne les prix, les normes de qualité, la fiabilité des livraisons tant au point de vue des délais que des quantités, ainsi que sur les conditions de paiement (possibilité de financement et d'échelonnement des règlements de factures). Par contre, dans des marchés où la vente implique de nombreuses interactions avec des clients dispersés, l'avantage revient à l'entreprise locale qui est mieux structurée pour répondre aux besoins particuliers de ce type de clientèle. Pour les marchés d'exportation, la petite entreprise locale ne pourra rivaliser ni avec la grande multinationale ni avec la petite firme à orientation mondiale car, pour ses ventes à l'étranger, elle devra s'en remettre à des agents ou à des importateurs souvent incontrôlables et très coûteux. De leur côté, les firmes mondiales chercheront, pour demeurer concurrentielles, à contrôler leurs réseaux de distribution, quitte à créer leurs propres circuits grâce à des filiales locales bien établies. 
En ce qui a trait au développement technologique, il y a lieu de distinguer la recherche et le développement sur les produits de ceux qui touchent les procédés. En effet, si les multinationales sont nettement favorisées par leur capacité de développement de nouveaux procédés, ces derniers requérant souvent d'importants investissements, il n'en est pas de même pour la conception de nouveaux produits où très souvent la PME innovatrice possède un avantage sur ses rivales. Par contre, même lorsque ces multinationales n'ont pas elles-mêmes mis au point un nouveau produit, c'est quand même à elles que l'on s'adresse pour leurs capacités d'application et de diffusion. Ces grandes entreprises ont donc accès à de nombreuses innovations technologiques même si elles ne sont pas le fruit de leurs propres recherches. Aussi, dans le domaine du développement technologique la situation est-elle nettement favorable aux grandes multinationales pour la recherche et le développement sur les procédés alors qu'elle tend à être équivalente pour la recherche et le développement sur les produits.

Finalement, en ce qui concerne les ressources financières, les entreprises multinationales ont généralement, grâce à leur capacité d'autofinancement, accès à des fonds à des coûts inférieurs à celui du marché. Elles peuvent notamment recycler leurs fonds là où les projets sont les plus prometteurs. De plus, elles ont facilement accès aux marchés financiers internationaux et elles jouissent généralement d'un traitement préférentiel de la part des institutions financières. Enfin, elles se voient souvent offrir par bien des gouvernements des incitations financières en contrepartie de l'établissement d'unités de production dans ces pays. Leurs activités mondiales leur permettent aussi de bénéficier de réduction d'impôts en négociant des régimes fiscaux favorables dans certains pays. Pour les PME mondiales, l'accès à des capitaux est peut être plus difficile, mais tout de même beaucoup plus aisé que pour une PME locale qui fait affaire avec des institutions financières souvent très réservées et prudentes lorsqu'il s'agit de structurer et de mettre en place des financements répondant à des besoins locaux.

Il y a lieu d'ajouter à ces quatre caractéristiques intrinsèques aux intervenants une dernière considération importante: le rôle des gouvernements. Leur attitude protectionniste nous amène à penser que sur un marché national les PME locales auront un avantage indéniable sur leurs rivales mondiales, bien que ces dernières puissent, elles aussi, comme nous l'avons vu précédemment, bénéficier dans certains cas de traitements de faveur des gouvernements des pays hôtes (subventions, barrières tarifaires ou régimes fiscaux avantageux).

Les divers avantages concurrentiels que possède l'entreprise mondiale sur sa rivale locale sont résumés au tableau suivant: 
Tableau 1

Les avantages concurrentiels respectifs des entreprises mondiales et des PME

\begin{tabular}{llll}
\hline Facteurs & FMN mondiales & PME mondiales & PME locales \\
\hline Production & Très favorisées & Défavorisées & $\begin{array}{l}\text { Nettement } \\
\text { défavorisées }\end{array}$ \\
Commercialisation & Favorisées & Favorisées & Défavorisées \\
Technologie & Très favorisées & Favorisées & Favorisées \\
Financement & Très favorisées & Favorisées & Défavorisées \\
Gouvernement & Défavorisées & Défavorisées & Très favorisées \\
\hline
\end{tabular}

Comme on pouvait s'y attendre, la situation est nettement à l'avantage des entreprises mondiales lorsqu'elles décident d'affronter une entreprise locale, car même sur son propre terrain, cette dernière ne peut résister bien longtemps. Il n'en reste pas moins que dans une évaluation beaucoup plus précise des menaces que représente la mondialisation, chacun de ces facteurs peut avoir plus ou moins d'importance selon la nature des activités de la PME considérée.

Pour procéder à une analyse plus poussée de l'impact de la présence d'une firme mondiale sur une PME, nous devons tenir compte de la position relative de ses activités vis-à-vis de celles de son homologue mondiale. Comme nous nous intéressons ici plus particulièrement aux PME confrontées à la présence d'une entreprise mondiale dans leur environnement immédiat, seules trois possibilités peuvent se présenter pour ces petites entreprises locales : être fournisseur, être distributeur ou être concurrent direct de la firme mondiale.

Dans le premier cas, celui de fournisseur d'une grande firme mondiale, la PME bénéficie de cet avantage confortable d'avoir des débouchés assurés pour sa production et des revenus garantis provenant d'un client bien établi. De plus, cette PME sera bien placée pour saisir les opportunités que lui présentera son client (développements technologiques conjoints, extensions des gammes de produits et déploiements géographiques). Ce noyau d'activités pourrait ainsi servir de base à la croissance rapide de l'entreprise. Toutefois, cette position présente aussi certaines menaces. Comme fournisseur captif d'une grande firme, la PME peut se voir rapidement confrontée à l'énorme pouvoir de négociation de son client qui aura très rapidement le dessus en l'obligeant à réduire ses prix et par le fait même sa rentabilité à défaut de quoi elle pourrait utiliser, grâce à son avantage technologique, des produits de substitution locaux ou d'importation. De plus, la PME pourrait craindre à plus long terme de voir l'entreprise mondiale, grâce à ses avantages de production et d'accès à des ressources financières, s'intégrer en amont soit localement ou dans un autre pays et mettre ainsi son avenir en péril. 
À titre de distributeur (une situation moins fréquente que la première), la PME pourra à court terme subir la pression de son fournisseur. En effet, les entreprises mondiales, en nombre restreint, dominent généralement leur marché. De plus, comme la PME ne représente probablement pas pour elle un client important, en augmentant ses prix elle réduira au minimum la marge bénéficiaire de la PME, qui à son tour ne pourra pas toujours charger la hausse de ses coûts d'approvisionnement à sa clientèle. La situation à long terme pourrait être beaucoup plus critique, car la PME aura toujours la crainte de voir l'entreprise mondiale désirer exercer un contrôle plus serré du marché. Comme nous l'avons vu précédemment, l'entreprise mondiale sera tentée pour des raisons stratégiques de se rapprocher du consommateur final et cherchera ainsi à procéder à une intégration verticale en aval. En outre, l'entreprise mondiale, désireuse d'écouler de gros volumes de production, risque aussi de se tourner vers de grandes chaînes de distribution nationales qui lui permettront d'accéder à un marché plus vaste. Ces situations ne s'appliquent pas aux PME actives dans des marchés fragmentés où la vente et le service exigent de nombreuses interactions avec des clients dispersés.

Dans le dernier cas, celui de concurrent direct de l'entreprise mondiale, la PME, comme nous l'avons vu dans la section précédente, ne possédant aucun avantage palpable sur ses grandes ou sur ses petites rivales mondiales risque, sans l'intervention de son gouvernement, de se voir éliminée rapidement du marché. C'est certainement la position stratégique la plus précaire et une des conséquences les plus néfastes du phénomène de la mondialisation pour les PME peu sensibles à ces enjeux.

Nous allons maintenant tenter d'établir, pour chacun de ces trois cas, les différentes options stratégiques offertes aux PME qui doivent faire face à la mondialisation.

\section{Les différentes options stratégiques envisageables pour les PME}

La grille d'analyse stratégique que nous allons utiliser reprend la typologie proposée par Noël (1989) pour étudier les stratégies des firmes entrepreneuriales. Elle comprend trois grandes catégories d'orientation stratégique que l'on retrouve dans la plupart des modèles de portefeuille (BCG, McKinsey et A.D. Little): le maintien, la croissance ou le retrait (Noël, 1990;1990b). Pour chacune de ces catégories, il existe différentes options stratégiques parmi lesquelles l'entreprise devra choisir. Le terme d'option stratégique s'apparente ici à celui de grande stratégie (Glueck, 1976) ou encore à celui de stratégie générique (Herbert et Deresky, 1987) et se réfère à certains choix stratégiques 
qui peuvent s'appliquer de manière générale quels que soient l'industrie, le type d'organisation ou sa structure et ce, afin d'en capturer l'essence tout en diminuant sa complexité. Cette grille stratégique comprend neuf options de base ${ }^{1}$ que nous devons, bien entendu, adapter aux PME. Une des caractéristiques fondamentales à évaluer est tout d'abord la propension de leurs dirigeants à coopérer avec d'autres entreprises ou, au contraire, leur préférence pour l'autonomie. Ainsi, nous avons choisi de regrouper certaines options pour mettre en évidence la propension à collaborer. Toutefois, certaines de ces options sont difficilement envisageables (acquisitions), car l'accès limité à des ressources financières constitue une des particularités de ces petites entreprises. Nous avons ainsi retenues, pour les PME, les six options stratégiques suivantes:

\subsection{Maintien}

\subsubsection{Maintien par ses propres moyens}

L'entreprise se contente, avec le minimum d'investissement, de maintenir ses activités dans leur état actuel et de ne faire que quelques petits ajustements pour diminuer ses coûts, améliorer sa situation financière ou corriger des défauts mineurs dans son fonctionnement. Si elle se sent menacée sur ses marchés, elle cherchera à contrecarrer les poussées de ses concurrents en mettant en place une série de mesures pour protéger son avenir, en tentant d'améliorer ses coûts et ses produits, en perfectionnant sa technologie ou en consolidant sa position sur le marché grâce à des interventions auprès de sa clientèle et de ses réseaux de distribution.

1. Ces neuf options stratégiques de bases sont les suivantes :

\section{Maintien}

1. Même taille, mêmes activités.

\section{Croissance}

2. Augmentation de la part de marché sur les marchés où l'entreprise est déjà présente.

3. Développement de nouveaux marchés.

4. Expansion des activités.

5. Croissance par fusion.

6. Croissance par acquisitions reliées.

7. Diversification non reliée.

\section{Désengagement}

8. Retrait avec démarrage d'une autre activité.

9. Retrait définitif. 


\subsubsection{Maintien avec recours à des ressources externes}

L'entreprise peut aussi défendre sa posture en faisant appel à des ressources externes. Pour cela, l'entreprise procède, grâce à des alliances stratégiques, à une mise en commun de certaines ressources avec d'autres firmes. L'alliance peut alors être verticale, si l'activité qui y est réalisée se situe en amont ou en aval de celles existant déjà chez les partenaires, ou horizontale, lorsqu'elle est similaire. Les alliances verticales ou complémentaires indiquent quant à elles une recherche d'expansion des activités des alliés en renforçant leurs habiletés sans pour autant modifier substantiellement leurs couples produit/marché d'origine. Les alliances horizontales ou supplémentaires indiquent la recherche d'un élargissement de leurs couples produit/marché en utilisant des habiletés transférables (Fernandez, 1993). Ainsi, l'entreprise pourra conclure soit une alliance complémentaire (verticale) pour renforcer certaines de ses activités, comme par exemple la mise au point d'améliorations technologiques de son procédé de fabrication ou de ses produits, soit une alliance stratégique supplémentaire (horizontale) pour augmenter l'envergure de ses activités. Ces associations peuvent prendre diverses formes juridiques allant de la mise en place d'un accord (de mise en marché, de production, de standardisation de produits, de service ou de développement technologique) à la formation d'une filiale commune (coentreprise ou partenariat).

\subsection{Croissance}

\subsubsection{Croissance interne}

Cette option s'adresse à une entreprise qui cherche à s'assurer une croissance plus rapide que son marché de base. Elle consiste en un développement graduel des activités de l'entreprise en misant sur ses compétences distinctives. L'entreprise pénètre au maximum son marché puis se tourne vers de nouveaux marchés qui, à leur tour, vont progressivement exiger une augmentation de sa capacité de production. La réalisation des trois étapes successives de ce processus, l'augmentation de la part de marché, suivie du développement de nouveaux marchés puis de l'accroissement de la capacité de production, est menée par l'entreprise seule, sans apport externe.

\subsubsection{Croissance externe}

Lorsque l'entreprise désire une croissance rapide, même si c'est parfois plus risqué, elle se tourne vers l'externe pour pouvoir avoir accès aux ressources d'autres entreprises (marché, capacité de production, technologie, capitaux, etc.). Cette option stratégique couvre une vaste gamme d'associations qui passe de 
l'alliance stratégique complémentaire ou supplémentaire sous ses différentes formes (simples accords ou coentreprises) à la fusion pour unir ses forces à une autre entreprise dans le cas d'entreprises dont les ressources et les capacités à prendre des risques sont limitées.

\subsection{Désengagement}

\subsubsection{Réorientation progressive vers d'autres secteurs}

Sans abandonner son noyau d'activités de façon soudaine, l'entreprise cherchera de nouveaux domaines et tentera de saisir les opportunités qui lui permettront de déplacer graduellement ses activités vers d'autres secteurs où son expertise l'aidera à développer de nouvelles compétences distinctives. Pour cela, elle pourra soit procéder à la mise au point à l'interne de nouvelles gammes de produits ou de services lui permettant d'effectuer un transfert progressif de ses activités à de nouveaux secteurs, soit faire appel à des ressources externes en s'associant avec d'autres firmes. Cette dernière approche consiste généralement à former une alliance stratégique complémentaire pour avoir accès à de nouveaux produits.

\subsubsection{Liquidation ou vente des activités}

En fonction de sa situation financière et en tenant compte des prérogatives de ses actionnaires, l'entreprise pourra soit, dans les cas extrêmes, être liquidée ou, dans de meilleures conditions, être cédée partiellement ou entièrement à un groupe d'intérêt qui pourra à son tour assurer sa pérennité.

Le tableau suivant résume les différentes options stratégiques que nous avons retenues pour une PME.

TABLEAU 2

Les différentes options stratégiques envisageables pour les PME

\begin{tabular}{lll}
\hline Options & Interne & Externe \\
\hline Maintien & $\begin{array}{l}\text { Amélioration } \\
\text { (coûts, produits, etc.) } \\
\text { Crónétration du marché, } \\
\text { développement de nouveaux } \\
\text { marchés, augmentation de } \\
\text { capacité, etc. }\end{array}$ & $\begin{array}{l}\text { Alliances stratégiques } \\
\text { ou fusion stratégiques }\end{array}$ \\
Désengagement & $\begin{array}{l}\text { Nouveaux produits, marchés } \\
\text { ou liduidation }\end{array}$ & $\begin{array}{l}\text { Alliances stratégiques } \\
\text { ou cession }\end{array}$ \\
\hline
\end{tabular}




\section{Les solutions retenues pour les PME confrontées à la mondialisation}

Pour chacune des trois positions relatives d'une entreprise locale vis-à-vis de sa rivale mondiale que nous avons relevées précédemment, nous pouvons à présent déterminer les différentes options stratégiques envisageables pour ces PME puis procéder à leur analyse afin d'évaluer celles qui répondent le mieux à la problématique posée par la mondialisation.

\subsection{Les solutions pour les fournisseurs d'une entreprise mondiale}

Pour le premier cas, celui de fournisseur, le choix d'options stratégiques pour la PME sera essentiellement limité à celles permettant à tout le moins de contrer, à court terme, les pressions de l'entreprise mondiale comme cliente et, à plus long terme, de pouvoir faire face à la menace d'intégration. L'entreprise peut raisonnablement envisager une des trois options stratégiques suivantes:

1. Le maintien avec, de préférence, un recours à des ressources externes, car cette option permet de mieux parer les risques encourus à court et à long terme et ce, en tenant compte des ressources limitées des PME.

2. La stratégie de croissance externe, car elle permet de matérialiser rapidement les occasions favorables qui peuvent se présenter et de résister aux menaces à court terme tout en permettant, dans certains cas, un désengagement plus rapide du secteur si les risques d'intégration de l'entreprise mondiale sont effectivement présents.

3. La réorientation progressive vers d'autres secteurs lorsqu'un désengagement est envisagé, car cette option présente tout au moins l'avantage de favoriser la survie à long terme de l'entreprise.

Parmi ces trois options stratégiques, nous devons à présent choisir celle qui non seulement répondra le mieux aux menaces posées par la présence d'une entreprise mondiale mais également qui améliorera la position de cette PME pour saisir des opportunités de croissance et qui, à notre avis, présentera le moins de difficultés pour sa mise en œuvre.

La première solution est celle du maintien avec, de préférence, un recours à des ressources externes. Comme nous l'avons vu précédemment, cette option consiste à mettre en place des alliances stratégiques. Cette solution a le mérite d'offrir une réponse adéquate et sur mesure aux problèmes créés par la mondialisation. En effet, l'alliance stratégique permet à une entreprise de défendre sa position stratégique contre des forces auxquelles elle ne pourrait résister seule et d'avoir accès à des ressources qu'elle ne pourrait acquérir autrement 
(Harrigan, 1986). Dans ce cas particulier, une alliance supplémentaire entre les différents fournisseurs géographiques d'une même entreprise mondiale peut leur donner conjointement un certain poids pour négocier avec ce client de taille mondiale. De plus, l'alliance stratégique peut être conçue de telle manière que des «clauses d'exclusivité» protègent chacun des partenaires dans son propre marché (McMillan, 1983). Des normes de produit peuvent aussi être uniformément établies, les améliorations technologiques des procédés et des produits, partagées et les renseignements techniques et commerciaux sur les fournisseurs, échangées entre les partenaires de l'alliance pour leur permettre de se protéger contre une éventuelle intégration en amont de l'entreprise mondiale.

Bien que l'alliance stratégique ait aussi le mérite de préserver jusqu'à un certain point l'indépendance de chacun de ses membres, elle présente par contre quelques inconvénients. Ses principales faiblesses résident dans sa mise en place et dans son bon fonctionnement. En effet, l'identification et le choix des bons partenaires, leur éventuelle contribution et leur type de structure organisationnelle peuvent créer quelques complications (Porter et Fuller, 1986; Bell, 1988; Main, 1990). De plus, son bon fonctionnement dépend de la capacité des associés à surmonter certaines difficultés comme l'importance des coûts de coordination, la compatibilité dans le temps des objectifs des alliés, l'établissement et le respect de frontières entre les partenaires, la confiance mutuelle, la complexité du contexte (différences de culture et de style de gestion), la création de valeurs et le maintien et la stabilité de l'alliance (Lorange et Probst, 1987; Olleros et McDonald, 1988; Koenig et Van Wijk, 1991). Bien que des problèmes de ce genre aient souvent mené à l'abandon et à la disparition précoces d'alliances stratégiques, il n'en reste pas moins qu'elles devraient fonctionner dans la mesure où les partenaires ont des tailles similaires et des objectifs communs. De plus, un climat d'entente et un état d'esprit favorisant la coopération devront s'établir entre ces entreprises et leurs dirigeants. Si ces difficultés peuvent être surmontées, l'alliance stratégique supplémentaire est une solution à retenir pour les PME faisant face au problème de mondialisation.

L'option de la croissance externe pour une PME consiste soit à la formation d'alliances stratégiques, soit à la fusion avec une autre entreprise. Comme nous l'avons vu auparavant, l'alliance stratégique supplémentaire devrait répondre convenablement aux problèmes de la mondialisation pourvu qu'elle soit mise en place de façon appropriée et que ses différents partenaires s'assurent de son bon fonctionnement. En outre, dans une optique de croissance externe, l'alliance permet d'accroître rapidement et à moindre coût les capacités d'une entreprise (Borys et Jemison, 1989) et de pénétrer rapidement de nouveaux marchés (Mowery, 1989). La fusion constitue une autre possibilité qui permet de répondre aux problèmes posés par la mondialisation. Son avantage réside avant tout dans le fait que ses effets et ses résultats sont quasi immédiats 
dans le contexte d'un marché mondial où une rapidité de réponse est essentielle. Par contre, son caractère irréversible ne laisse que peu de marge de manœuvre en cas d'erreur. Enfin, c'est une solution souvent difficilement envisageable pour des PME où la propriété est entre les mains des dirigeants seulement.

Par conséquent, ce type de solution s'adressera à des entreprises possédant de nombreuses affinités. En effet, leur mariage doit se faire tant au niveau des dirigeants, qui devront être prêts à partager leur pouvoir et faire d'importantes concessions et de nombreux compromis, qu'à celui des organisations qui devront adapter leur structure et leur méthode de fonctionnement l'une à l'autre (Shrivastava, 1986). Le caractère délicat d'une telle transaction est d'autant plus crucial que si l'on ne parvient pas à surmonter certaines de ces difficultés, les deux partenaires seront du même coup pénalisés et l'objectif même de cette stratégie sera gravement compromis. Cette solution pourrait donc être sérieusement envisagée par deux partenaires qui ont réussi à mettre en place une alliance stratégique et à cohabiter avec succès et qui désirent poursuivre leur coopération de façon encore plus étroite.

La dernière solution envisageable est celle qui consiste à réorienter progressivement les activités de l'entreprise. Elle a le mérite de ne faire appel qu'à ses ressources internes (son expertise). Cette solution permet ainsi à la PME et à ses dirigeants de garder le contrôle du processus de mise en œuvre de la réorientation avec un minimum d'interférences externes. De nombreuses PME, qui sont en majorité des entreprises à structure simple (Mintzberg, 1979) ayant à leur tête un propriétaire prenant toutes les décisions, préféreront cette solution. Cet entrepreneur utilisera tous ses talents et toute son énergie pour repérer les possibilités d'affaires s'offrant à son entreprise et en tirer profit. Même si cette solution recevra rapidement l'adhésion du dirigeant de l'entreprise, ses inconvénients sont nombreux. D'une part, la mise en œuvre d'une telle stratégie dépendra des ressources internes d'une entreprise de petite taille qui seront fort probablement très limitées. D'autre part, le changement des activités de l'entreprise dépendra entièrement des capacités d'une seule personne, son propriétaire, de ses états d'âme et de sa faculté à identifier et à analyser de nouvelles opportunités. De plus, l'entrepreneur qui mettra cette stratégie en œuvre sera amené à prendre de nombreuses décisions qui exigeront une très bonne connaissance des limites des compétences de son organisation et de sa compréhension de la dynamique concurrentielle des nouveaux créneaux auxquels il s'attaquera. Finalement, cette option ne résoud pas complètement les problèmes à court terme posés par la mondialisation. En effet, l'entreprise mondiale exercera des pressions pour réduire la rentabilité de la PME dont la structure centralisée ne fera que ramener très rapidement toute cette problématique au niveau du dirigeant. Celui-ci risque de voir son attention détournée de la mise en œuvre de sa stratégie qui sera par le fait même sérieusement compromise. 
La mise en place d'alliances supplémentaires semble donc la solution la plus appropriée, bien qu'une fusion avec une autre PME pourrait aussi dans certains cas être envisagée.

\subsection{Les solutions pour les distributeurs de produits mondiaux}

En ce qui a trait au second cas, celui de distributeur, les menaces sont essentiellement les mêmes que celles du cas précédent: pressions à court terme menant à une réduction de la marge bénéficiaire de la PME, mais avec une menace à plus ou moins long terme beaucoup plus sérieuse d'intégration verticale en aval, car l'entreprise mondiale cherchera à se protéger en se rapprochant de la clientèle. Il n'en reste pas moins que les options stratégiques que l'on peut raisonnablement envisager sont essentiellement, et pour les mêmes raisons, identiques à celles du premier cas :

- le maintien avec, de préférence, un recours à des ressources externes;

- la croissance externe;

- la réorientation progressive menant tôt ou tard au désengagement.

Comme ces solutions sont semblables à celles du cas précédent, les mêmes conclusions s'imposent. Toutefois, dans le cas d'une PME essentiellement engagée dans la distribution, une réorientation des activités vers d'autres domaines connexes semble beaucoup plus facile que dans le cas précédent. En effet, les actifs tangibles de l'entreprise sont généralement plus légers, ce qui rend leur redéploiement vers d'autres produits et services plus rapide et moins coûteux, dans la mesure où les marchés desservis restent les mêmes. Cette réorientation des activités de l'entreprise vers d'autres secteurs consistera à rechercher au moyen d'alliances complémentaires, de nouveaux fournisseurs et, par conséquent, de nouveaux produits à distribuer. Cette solution offre donc une réponse au moins tout aussi intéressante au problème à court terme que l'alliance supplémentaire. De plus, comme le partenariat d'une alliance complémentaire est composé de firmes non concurrentes, sa mise en place et son bon fonctionnement devraient être plus simples et plus rapides. Ces raisons font que nous préférons cette solution à l'alliance stratégique supplémentaire ou à la fusion.

\subsection{Les solutions pour les concurrents directs d'entreprises mondiales}

Pour ce qui est du dernier cas, celui de concurrent direct d'une grande entreprise mondiale, la seule option envisageable est un désengagement aussi rapide que possible du marché, pour pouvoir procéder dans les conditions les plus avantageuses à la vente de l'entreprise et dans le meilleur des cas à sa cession à une entreprise mondiale peu après que celle-ci aura fait son entrée sur son marché. 
Si ce n'est pas possible, un retrait progressif, avec une diminution des activités de l'entreprise jusqu'à sa fermeture ou sa liquidation, devrait être considéré.

Par contre, dans les cas de micro-marchés, il est fort probable que la rivale mondiale soit, elle aussi, une petite ou moyenne entreprise; les options envisageables pour une PME locale qui désire protéger son marché sont alors le maintien avec un recours à des ressources externes ou la croissance si elle préfère affronter de manière plus énergique sa concurrente mondiale. Par conséquent, la croissance externe par le biais d'alliances devrait être envisagée, car cette solution facilitera un déploiement rapide sur de nombreux marchés et permettra ainsi de contenir la progression de la concurrente mondiale. En fait, cette solution consiste à «mondialiser» le plus rapidement possible les activités de la PME. La croissance interne peut aussi être considérée, mais comme elle est moins rapide et exige plus de ressources, c'est donc une solution moins avantageuse que le recours à des alliances.

\subsection{Les solutions retenues}

Nous pouvons à présent dresser un tableau récapitulatif des hypothèses de solutions à retenir pour les PME qui font face aux problèmes engendrés par la mondialisation:

TABlEAu 3

Les solutions à la mondialisation

\begin{tabular}{|c|c|c|c|c|}
\hline & \multicolumn{4}{|c|}{ Position de la PME vis-à-vis l'entreprise mondiale } \\
\hline & Fournisseur & Distributeur & $\begin{array}{l}\text { Concurrent } \\
\text { direct d'une } \\
\text { GE mondiale }\end{array}$ & $\begin{array}{l}\text { Concurrent } \\
\text { direct d'une } \\
\text { PME mondiale }\end{array}$ \\
\hline $\begin{array}{l}\text { Stratégie } \\
\text { préférée }\end{array}$ & $\begin{array}{l}\text { Alliance } \\
\text { stratégique } \\
\text { supplémentaire }\end{array}$ & $\begin{array}{l}\text { Alliance } \\
\text { stratégique } \\
\text { complémentaire }\end{array}$ & $\begin{array}{l}\text { Vente de } \\
\text { l'entreprise }\end{array}$ & $\begin{array}{l}\text { Alliances } \\
\text { stratégiques }\end{array}$ \\
\hline $\begin{array}{l}\text { Stratégie } \\
\text { envisageable }\end{array}$ & $\begin{array}{l}\text { Fusion avec } \\
\text { PME compatible }\end{array}$ & $\begin{array}{l}\text { Alliance } \\
\text { stratégique } \\
\text { supplémentaire }\end{array}$ & $\begin{array}{l}\text { Retrait } \\
\text { progressif } \\
\text { liquidation }\end{array}$ & $\begin{array}{l}\text { Croissance } \\
\text { interne }\end{array}$ \\
\hline
\end{tabular}

Les solutions présentées ci-dessus ne sont pas mutuellement exclusives. Il existe en fait de nombreuses stratégies intermédiaires parmi lesquelles l'entreprise pourrait choisir. Les options stratégiques préférées et envisageables devraient plutôt servir de points de repère aux PME concernées par la mondialisation et les aider à formuler leur propre stratégie en fonction de leurs particularités. Pour les chercheurs, elles présentent des cas types à explorer, décrire et évaluer. 


\section{Conclusion}

L'analyse du phénomène de la mondialisation nous indique clairement que la proximité d'entreprises mondiales peut engendrer certaines menaces pour les PME locales, notamment celles des secteurs les plus vulnérables de notre économie ou, encore, les plus en croissance sur le plan mondial. Comme notre infrastructure industrielle est composée en majorité de PME créatrices d'emplois, il est essentiel de se pencher rapidement sur l'identification et la mise en place de mesures qui permettront d'améliorer leur compétitivité pour non seulement défendre leurs marchés locaux, mais aussi pour pouvoir exporter vers les marchés internationaux. Notre recension des recherches nous a montré qu'il n'existe pas, à première vue, de solution miracle au problème de la mondialisation pour ces petites entreprises. Par contre, plusieurs options stratégiques peuvent être envisagées et celles que nous avons retenues offrent, suivant notre compréhension des recherches antérieures, les meilleures chances de réussite et méritent qu'une PME menacée par la présence d'une firme mondiale dans son environnement immédiat les étudie de façon prioritaire. Parmi les solutions que nous avons retenues, pour une étude empirique ultérieure, l'alliance stratégique présente un potentiel intéressant qui mérite une analyse plus approfondie (Noël et Zhang, 1992, 1993). Par contre, certaines particularités des PME, comme leur profond désir d'indépendance et leur besoin de flexibilité, rendent sa mise en œuvre ardue et parfois impossible. Il serait donc particulièrement important de déterminer dans ces petites entreprises les conditions qui facilitent la mise en place d'alliances, les facteurs qui favorisent son évolution vers le succès ainsi que les paramètres de politique industrielle qui peuvent encourager le recours à cette solution. L'utilité d'une telle démarche nous semble fondamentale, car les PME n'ont pas les ressources pour se doter de stratégies pouvant les aider à affronter la problématique créée par la mondialisation et pour s'intégrer dans la nouvelle dynamique de marché qu'engendre l'émergence des régions avec des traités comme celui de libre-échange avec les États-Unis et le Mexique. Ces entreprises ont un besoin pressant d'informations pratiques, de formation de leurs gestionnaires et de soutien pour mettre en œuvre des solutions qui pourront assurer leur survie et favoriser leur croissance. Quant aux chercheurs, il devient urgent de canaliser leurs recherches en stratégie pour décrire et mesurer les effets des options envisageables pour les PME qui voudraient survivre, sinon profiter de la mondialisation pour améliorer leurs contributions à la société. 


\section{Bibliographie}

BELL, J. (1988), «The winning organization», Fortune, 26 septembre, p. 50-60.

BORYS, B. et D.B. JEMISON, (1989), «Hybrid arrangements as strategic alliances : theoretical issues in organizational combinations », Academy of Management Review, vol. $14, \mathrm{n}^{\circ} 2$, p. 234-249.

ChANDLER, A.D. (1986), «The evolution of modern global competition», dans M.E. Porter (dir.), Competition in Global Industries, Boston, Harvard Business School Press, p. 405-448.

FERNANDEZ, G.P. (1993), «Les alliances stratégiques », Montréal, Cahier d'enseignement du CETAI 93-01, École des HEC.

GlueCK, W.F. (1976), Business Policy: Strategy Formation and Management Action, New York, McGraw-Hill.

HARRIGAN, K.R. (1986), Managing for Joint Venture Success, Lexington, D.C. Heath and Company.

HERBERT, T.T. et H. DERESKY (1987), «Generic strategies : an empirical investigation of typology validity and strategy content», Strategic Management Journal, vol. $8, \mathrm{n}^{\circ} 2$, p. 135-147.

KoeniG, C. et G. VAN WIJK (1992), «Alliances interentreprises : le rôle de la confiance », dans Alain NoËL, Perspectives en management stratégique, Tome 1, Paris, Economica, p. 305-327.

LORANGE, P. et G.J.B. PROBST (1987), « Joint ventures as self-organizing systems : a key to successful joint venture design and implementation», Columbia Journal of World Business, été, p. 71-77.

MAIN, J. (1990), «Making global alliances work», Fortune, 17 décembre, p. 121-126

MCMillan, I.C. (1983), «Preemptive strategies », The Journal of Business Strategy, vol. $4, \mathrm{n}^{\circ} 2$, p. 16-26.

MERRIFIELD, B. (1989), «Strategic alliances in the global market place », Research Technology Management, janvier-février, p. 15-20.

MINTZBERG, H. (1979), The Structuring of Organizations : A Synthesis of the Research, Englewood Cliffs, N.J., Prentice-Hall.

MOWERY, D.C. (1989), «Collaborative ventures between U.S. and foreign manufacturing firms », Research Policy, 18.

NOËL, A. (1989), Entrepreneurship and Strategies for the EEC of 1992 : An Exploratory Study, Montréal, Cahier du CETAI 89-01, École des HEC.

NoËL, A. (1990), «Growing through alliances: who goes for it and who doesn't ?», Montréal, Cahier du CETAI 90-12, École des HEC. 
NoËL, A. (1990b), «Les entreprises québécoises face à la mondialisation: la voie des alliances », Montréal, Gestion, vol. 15, n 3, École des HEC, p. 46-58.

NoËL, A. et J. ZHANG (1993), «Alliances stratégiques : une bibliographie thématique », Montréal, Cahier de recherche du CETAI 93-06, École des HEC.

NoËL, A. et J. ZHANG (1992), «Alliance stratégique et stratégie d'alliance », Montréal, Cahier de recherche du CETAI 92-15, École des HEC.

Olleros, F.J. et R.J. MACDONALD, (1988), «Strategic alliances: managing complementarity to capitalize on emerging technologies», Technovation, vol. 7, $\mathrm{n}^{\circ} 2$, p. 155-176.

Ohmae, K. (1985), Triad Power : The Coming Shape of Global Competition, New York, The Free Press.

PICKHolz, J.W. (1988), «The end of the world (as we know it) : market segmentation is changing the way the world turns », Direct Marketing, septembre, p. 42-45.

PORTER, M.E. (1986), Competition in Global Industries, Boston, Harvard Business School Press.

PORTER, M.E. et M.B. FUller (1986), «Coalitions and global strategy», dans M.E. Porter (dir.), op. cit., p. 315-344.

ShrivaSTAVA, P. (1986), «Postmerger integration», The Journal of Business Strategy, p. 65-76. 